\title{
Correction to Black Hole Entropy Variation under Hawking Radiation
}

\author{
Shad Ali, ${ }^{1,2}$ Misbah Ullah, ${ }^{3}$ and Johar Zeb ${ }^{4}$ \\ ${ }^{1}$ Department of Physics, University of Okara, 56300, Punjab, Pakistan \\ ${ }^{2}$ Department of Astronomy, Xiamen University, Xiamen, Fujian 361005, China \\ ${ }^{3}$ Department of Center for Nanosciences, University of Okara, 56300, Punjab Pakistan \\ ${ }^{4}$ Department of Physics, Beijing Normal University, Beijing, 100875, China
}

\begin{abstract}
We discussed some feature and facts associated to black hole and investigated the process of black hole evaporation by using the concept of interior volume and entropy. For this purpose, the maximal interior volume and quantum modes entropy of Kerr Newman Black Hole $(\mathrm{KNBH})$ is found to be linearly growing with Eddington time $\tau$. Which means that the thermodynamical quantities in the interior of black hole are changing with time and information can be restored in its interior. Considering two modified assumptions, an evolution relation is obtained between the entropy of $\mathrm{KNBH}$ with Bekenstein Hawking entropy. It gives a good insight for understanding the black hole evaporation process under Hawking radiation. In addition, these analysis also generalizes the characteristic features of black hole evaporation.
\end{abstract}

Keywords: black hole as black body (NPBB), interior volume, entropy, Hawking radiations, evaporation DOI: 10.31526/ACP.BSM-2021.36

\section{INTRODUCTION}

The discovery of Hawking radiation and its adjustment with quantum theory has been made in fast several decade but its unitary evolution is inconsistent with quantum theory in the final stage of black hole evaporation. As the information after evaporation is not conserved. This topic is given the name of black hole Information Paradox [1]. Several attempts were made to resolve this issue but still no satisfying result is obtained. It is accepted that black hole preserves the information and several models are offered to explain the process of information loss form black hole from initial stage to final stage. The quantum gravitational effects are considered to have vital role in this process [2]. Here, we will evolve the black hole interior informations and possible solution of information Paradox using the concept of black hole interior volume and entropy to generalize the emission process.

In general relativity the concept of black hole interior volume is a challenging problem due to choice of hyper-surface and interchange of space time coordinates in the interior of black hole. Parikh [3], found the interior volume of a stationary black hole as slicing invariant. Recently, Christodoulou and Rovelli (CR) [4,5] stated that there exists a maximal space-like hyper-surface bounding the largest interior volume of the black hole, which increases with advance time $v$. It has been. So, there is enough room to store the information in it. This means that the smallness of black hole size is misunderstanding of black hole interior volume.

Following the CR work of interior volume, Baocheng Zhang [6] investigated the interior quantum modes entropy of black hole by a semi-classical way, which showed that the interior quantum modes entropy of black hole also increases with advance time. As the black hole volume and entropy are found increases with advance time. So, this relation of advance time with interior quantum modes entropy may be a candidate to affect the statistical quantities. Using two assumptions, an evolution relation is obtained between the interior and exterior entropy and important discussion is contributed to the literature of black hole physics for detail also see Refs. [10, 11, 12]. Here are some points, which need attention to make some advancement in above discussion.

If we think of general result in this literature here a black hole is considered as an isolated system, i.e. in most cases the black holes solution is carried out for Einstein field equation in vacuum state by using static, static charged, rotating or charged rotating solutions. Note from an isolated system, we means black hole as a system with no external effects on it. Whereas in actual situations there exists other astronomical bodies that are directly attracted with black holes. Like in BH-BH or BH-NS merging process a Black hole/neutron star is attracted with black hole to produce FRBs and GRBs as result of disturbance in Magetohydrodynamics wind, which result in the emission of gravitational waves. According to Stephan Hawking work, black hole is a thermodynamic object by emitting Hawking radiations. These radiations can be evolved to get the emission process, but this is a special case for the emission of radiation from black hole. In general, a black hole absorbs all type of radiations and one could consider it as gray body. By doing so, here want to summarize some facts/results to understand the notion of black hole as gay body. The main aspects which contradicts the existence of black body in nature of black hole are as under:

1. As from black hole theories, a black hole is defined by space-time metric. Which describe an isolated space-time. While as a part of our universe, they are non-isolated.

2. Classically, a black hole swallow everything from its surroundings entering to its horizon and can't escape out of it due to its strong gravitational forces. Whereas quantum mechanically, black hole has the property of virtual pairs creation and 
evaporation as discussed by Hawking [14, 2]. If black hole emits Hawking radiations, then necessarily, these radiations are black body radiations or an approximate to it?

3. The most important point for difference between the two objects is that the emission of radiation from black hole increases with time and finally evaporates having an infinite temperature. Whereas, the emission of radiation from black body decreases with time until its temperature is adjusted with surrounding temperature. Next, A black hole evaporates due to quantum effects on its horizon, which leads to emission of radiation as claimed by Hawking, whereas black body only emits radiations/energy, which it has been absorbed.

If one consider black hole as perfect black body (PBB), then these aspects will be effective on the precision of results of black hole evaporation and considering black hole as PBB becomes a special case for the emission of black body radiation. So, we need a flexible notion to reveal the validation of emission process. The most exquisite idea for overcoming the above aspects is to introduce an emissivity factor $\epsilon$ with values $0 \leq \epsilon \leq 1$. Where $\epsilon=0$ represent null emission rate and $\epsilon=1$ represent black hole as PBB with highest emission rate. Using this idea, we can extend our final result to either black hole as PBB or nearly perfect black body (NPBB). For this purpose, we need to modify the two assumptions that satisfy our result in flexible form. These two assumptions will be discussed in the next section. The main advantage of this work will help us to get the emission rate in its actual form without losing the Physics of radiation emission.

By using the above notion, we will investigate the black hole evaporation under Hawking radiation. The organization of this paper is such that in next sect. 2, we will investigate the interior volume bounded by the largest space-like hyper-surface and interior entropy of its scalar quantum modes. In sect. 3, following two important assumptions to get an evolution relation between the interior quantum modes entropy and Bekenstein entropy. This proportional relation is more reliable due to varying value of emissivity factor. The final sect. 4 , is devoted to results and discussion.

\section{INVESTIGATION OF ENTROPY VARIATION AND EVAPORATION UNDER HAWKING RADIATION}

Let us consider Kerr Newman (KN) black hole, whose metric in Eddington Finkelstein coordinates can be written as:

$$
d s^{2}=\frac{\left(\Delta-a^{2} \sin ^{2} \theta\right)}{\rho^{2}} d v^{2}+\rho^{2} d \theta^{2}+2 d v d r-\frac{2\left(2 m r+Q^{2}\right)}{\rho^{2}} a^{2} \sin ^{2} \theta d v d \phi-2 a \sin ^{2} \theta d r d \phi+\frac{A \sin ^{2} \theta}{\rho^{2}} d \phi^{2}
$$

where

$$
\Delta=r^{2}-2 M r+a^{2}+Q^{2}, \quad \rho^{2}=r^{2}+a^{2} \cos ^{2} \theta, \quad A=\left(r^{2}+a^{2}\right)^{2}-a^{2} \Delta \sin ^{2} \theta, \quad J=M a
$$

$(J, Q)$ are the angular momentum and charge for unit mass of black hole satisfying the condition $M^{2}>a^{2}, \quad M^{2}>Q^{2}$. The vanishing value of $\Delta$ gives the positions of interior and exterior horizons as:

$$
r_{ \pm}=M \pm \sqrt{-a^{2}+M^{2}-Q^{2}}
$$

these surfaces are referred to as $r_{+}$and $r_{-}$horizons. The former one is called the event horizon and the latter on is called the Cauchy horizon. Whereas the position these two horizons $r_{+}<r<r_{-}$is referred to as the ergo-sphere. The interior volume discussed in [7], can be expressed as

$$
V=2 \pi f[M, Q, a] v
$$

Where

$$
f[M, Q, a]=\sqrt{-\Delta}\left(\sqrt{a^{2}+r^{2}}+\frac{r^{2}}{2 a} \log \left(\frac{\sqrt{a^{2}+r^{2}}+a}{\sqrt{a^{2}+r^{2}}-a}\right)\right)_{r \rightarrow r_{\max }}
$$

and $v$ is termed as Eddington time or advance time. The function $f[M, Q, a]$ has its maximum value $f_{\text {max }}$ for the spatial coordinate $r \rightarrow r_{\max } \approx 1.3005$ as shown in the Fig. (1) below. The proportional relation between interior volume and advance time in Eq. (3) is the special character of black hole interior volume.

According to Ref.[13, 4], the entropy in the interior volume of black hole can be generally defined as

$$
S=\frac{\pi^{2}}{45 \beta^{3}} V_{\max }=\frac{2 \pi^{3} f_{\max }}{45 \beta^{3}} v
$$

where $f_{\max }$ is the maximum value of $f[M, Q, a]$ and

$$
\beta=T^{-1}=\frac{2 \pi\left(2 M\left(M+\sqrt{M^{2}-a^{2}-Q^{2}}\right)-Q^{2}\right)}{\sqrt{M^{2}-a^{2}-Q^{2}}}
$$

is the inverse Hawking temperature of Kerr Newman black hole. As in Eq. (4), the entropy ib the interior of KNBH is also related to the Eddington time, so the property of Eddington time may be treated to affect the statistical quantities in the interior of black 


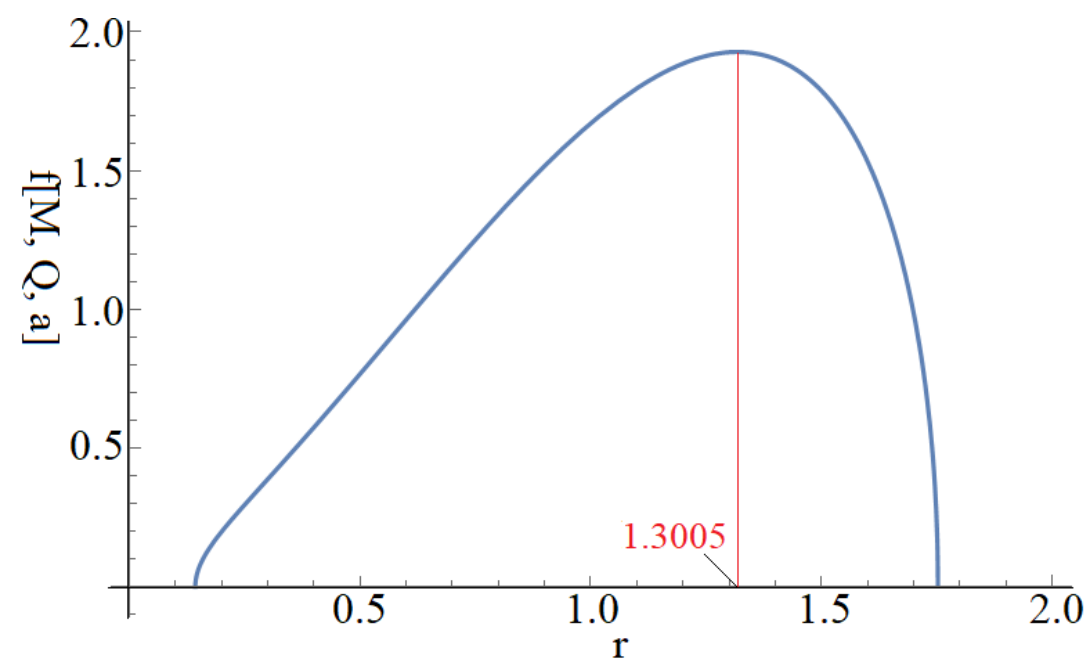

FIGURE 1: Plot of $f[M, Q, a]$ vs. $r$ of Kerr Newman black hole showing the position of maximal hyper surface.

hole for a possible solution of the problem of information paradox. Using this fact, we can prob this special case of statistical quantum modes entropy with the horizon entropy to see the effect of the two entropy and its relation with information paradox. Fortunately, it is stated in Ref. [3], that the relation between the two entropies is the reflection of black hole information paradox. For this purpose, we will follow the technique of black hole evaporation via emission of radiation.

\section{EVOLUTION OF PROPORTIONAL RELATION BETWEEN THE TWO ENTROPY}

Up to now, using interior quantum modes in a scalar field of black hole, we have been investigated the entropy in the interior of black hole is directly proportional to the Eddington time. Using the property of advance time as main factor of affecting the statistical quantities in the interior of black hole. So, let us consider the change in entropy and involve the emission of radiation in our process of investigations. For this purpose, let we introduce two modified assumptions for radiation emission from black hole as

- Black hole radiations as NPBB radiations: This assumption could guarantee the black hole temperature seen from infinity as event horizon temperature so, one can use the Boltzmann law for the emission of radiation $[6,10]$. In $(3+1)$ dimension space-time of rotating BTZ black hole the Boltzmann law can be written as:

$$
\partial_{\nu} M=-\sigma \epsilon A T^{4}+\frac{Q r_{+}}{r_{+}^{2}+a^{2}} \partial_{\nu} Q+\frac{a}{a^{2}+r_{+}^{2}} \partial_{\nu} J, \quad \epsilon=\frac{M}{M_{o}}
$$

Here $A=4 \pi\left(r_{+}^{2}+a^{2}\right)$ is the area and $T=\frac{1}{4 \pi} \frac{r_{+}-r_{-}}{r^{2}+a^{2}}$ is the horizon temperature, $M$ is the mass of of KN black hole acting as $\mathrm{NPBB}$ and $M_{o}$ is the same mass of PBB at the same temperature as $M$. For $M=M_{o} \Rightarrow \epsilon=1$ one gets Boltzmann equation for an ideal black body. In this equation, the second and third terms are the radiation powers (correction term) due to angular momentum and charge. Using the values of $A, T$ and $r_{+}$, we get this equation as:

$$
\partial M=\left(-\frac{\left(r_{+}-r_{-}\right)^{4} \sigma \epsilon}{64 \pi^{3}\left(r_{+}^{2}+a^{2}\right)^{3}}+\frac{a}{r_{+}^{2}+a^{2}} \partial_{\nu} J+\frac{Q r_{+}}{r_{+}^{2}+a^{2}} \partial_{\nu} Q\right) \partial \nu
$$

As our investigations are based on the assumption of $v>>M$ from which the extended result in term of charge and angular momentum becomes as $M>>Q \Rightarrow \partial_{\nu} Q<<1$ and $M>>J \Rightarrow \partial_{\nu} J<<1$. This means that the contribution of charge and angular momentum to the emission of radiation rate are negligible. So, in latter cases, we will not consider these quantities for accounting the emission of radiation. So, the above equation becomes:

$$
\partial M=-\frac{\sigma \epsilon\left(M^{2}-a^{2}-Q^{2}\right)^{2}}{4 \pi^{3}\left(2 M\left(\sqrt{M^{2}-a^{2}-Q^{2}}+M\right)-Q^{2}\right)^{3}} \partial \nu
$$

- Considering the emission rate as quasi-static i.e. $\partial_{v} M<<1$. which means the evaporation process is slow enough but, Hawking temperature is changing. Due to this assumption the thermal equilibrium between the scalar field inside black hole and the event horizon is preserved in an adiabatic process. This assumption could guarantee us the differential form for infinity small interval of time. 
So, the differential form of interior quantum modes entropy as:

$$
d S=\frac{1}{180}\left[X(M, Q, a)+v \partial_{M} X(M, Q, a) \partial_{v} M+v \partial_{Q} X(M, Q, a)+v \partial_{a} X(M, Q, a) \partial_{d v} a\right] \partial v \approx \frac{1}{180}[X(M, Q, a)] \partial v
$$

Where

$$
X(M, Q, a)=\frac{f_{\max }\left(M^{2}-Q^{2}-a^{2}\right)^{3 / 2}}{\left(2 M^{2}-Q^{2}+2 M \sqrt{M^{2}-Q^{2}-a^{2}}\right)^{3}}
$$

Now, consider the Bekenstein Hawking entropy is defined as:

$$
\left.S_{B H}=\frac{A}{4}=\pi\left(2 M\left(M+\sqrt{M^{2}-a^{2}-Q^{2}}\right)-Q^{2}\right)\right)
$$

Its differential form can be written as:

$$
d S_{B H}=\frac{2 \pi\left(M+\sqrt{M^{2}-a^{2}-Q^{2}}\right)^{2}}{\sqrt{M^{2}-a^{2}-Q^{2}}} d M
$$

Using modified form of Stephan Boltzmann law from Eq. (8) and ignoring the effect of charge and angular momentum the generalized result be can written as:

$$
d S_{B H}=-\frac{\sigma \epsilon}{2 \pi^{2}} Y(M, Q, a) d v
$$

Where

$$
Y(M, Q, a)=\frac{\epsilon\left(M^{2}-a^{2}-Q^{2}\right)^{3 / 2}}{\left(2 M\left(M+\sqrt{M^{2}-a^{2}-Q^{2}}-Q^{2}\right)\right)^{2}}
$$

Using the restriction on charge and angular momentum, the proportional relation between the differential forms of scalar field entropy and Bekenstein-Hawking entropy for an infinitesimal interval of time can be expressed as:

$$
\frac{d S}{d S_{B H}}=-\frac{\pi^{2}}{90 \sigma \epsilon}\left[\frac{X(M, Q, a)}{Y(M, Q, a)}\right]
$$

In general form this evolution relation between scalar field entropy and Bekenstein-Hawking entropy under Hawking radiation can be expressed as:

$$
d S=-\frac{\pi^{2}}{90 \sigma} F(M, a, Q) d S_{B H}
$$

where

$$
F(M, Q, a)=\frac{f_{\max }(M, a, Q)}{\epsilon\left(2 M\left(M+\sqrt{M^{2}-a^{2}-Q^{2}}\right)-Q^{2}\right)}
$$

Considering modified Stephan Boltzmann law in Eq. (8), we get the above Eq. (16). If the emissivity factor $\epsilon=1$ then this proportionality relation is same as that for the black hole as a black body. For black hole to be nearly perfect black body, the emissivity factor $\epsilon$ is always less than 1 . So, we can use this relation of black hole evaporation for emissivity ranging $0<\epsilon \leq 1$.

The plot in Fig. (2) shows that increasing or decreasing the emissivity factor lead to greatly affects on the emission of radiation from black hole. This result generalizes our earlier study of black hole evaporation [11, 12]. In comparison to this result, our earlier investigations for black hole as black body can be considered as a special case of this study. The special character of this result is the effects of emissivity factor in the evolution process of black hole. This result could describes the evaporation of black hole even if the black hole is not a PBB.

\section{RESULTS AND DISCUSSION}

Following the work stated in Refs. [4, 7], the interior volume of KN black hole is found to increase directly with Eddington time. Next, the scalar field of black hole interior quantum mode is taken in account for investigation of entropy. It is found proportional to Eddington time. This characteristic feature of advance time gives a hope for changing the thermodynamic properties in the interior of black hole. As several contradictions for black hole as PBB are noted for possible amplification. So, we considered two modified assumptions of black hole as NPBB and the radiation emission rate as quasi-static as discussed in Sec. 2. These two assumptions led us to consider horizon temperature as Hawking temperature and compute its interior quantum modes entropy in differential form. According to Parikh [3] statement for the possible bound between interior quantum modes entropy and Bekenstein Hawking entropy, we investigate a proportional relation between the two entropy by using the Stephan Boltzmann law and its plot is shown below in Fig. (2). 
0.3

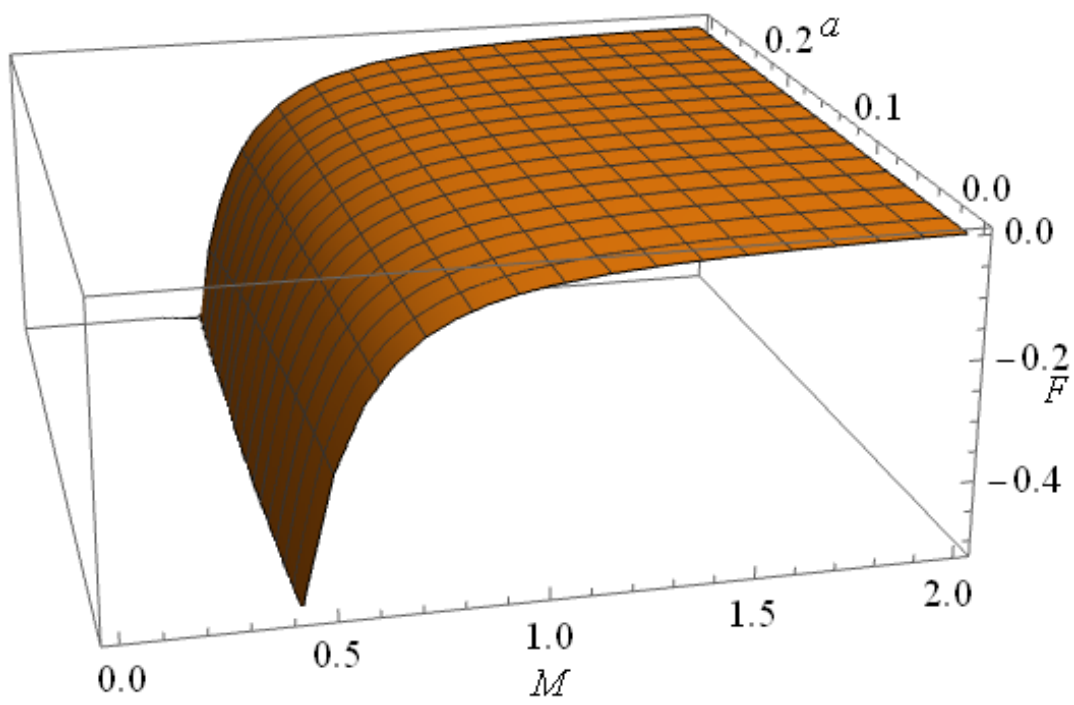

FIGURE 2: Plot of $M$ and a versus $F(M, Q, a)$ for Kerr Newman black hole radiations acting NPBB radiations. Here $M<M_{0}$.

The curve of this plot in three parts as under: The first part of this curve seems as flat, which shows the emission rate at this stage is slow enough. It gradually increases with time whereas the mass decreases. The second part is the turning part, showing that the emission rate of black hole increases and in final stage the evaporation process has its peak value (third part). The black hole temperature at final stage is also high enough. Using the emissivity factor $\epsilon$, we evaluate the emission rate for the black hole at different natures, i.e. introducing the emissivity factor in emission process, which gives a good result even if the black hole is not a perfect black body. Moreover, the contribution of charge $Q$ and angular momentum $J$ to the emission rate of black hole is negligible or more generally, one can consider the angular momentum as conserved quantity as seen from angular momentum line in Fig. (2)

This evolution relation for $\epsilon=1$ is found to satisfy the earlier result of black hole as PBB with highest emission rate. As long as its value is $0<\epsilon \leq 1$ the black hole acts as NPBB. Similarly, by increasing the value of emissivity factor form zero onward, the area of the plot increases and at $\epsilon \rightarrow 1$ it has maximum area. It seems that considering the emissivity factor in black hole emission process may change the results of black hole evaporation if the black hole is not PBB. Moreover, considering different emissivity factors, one could claim the difference in nature and properties of black holes. Whereas the black hole as PBB under Hawking radiation can be seen as a special case of this study. Which can be degenerated from this result by taking unit emissivity factor i.e. $M_{o}=M$.

\section{ACKNOWLEDGMENTS}

This work is supported by National Academy of Higher Education, High Education Commission of Pakistan (Grant No. 7/IPFPII(Batch-I)/SRGP/NAHE/HEC/2020/114).

\section{References}

[1] S. W. Hawking, "Breakdown of Predictability in Gravitational Collapse," Phys. Rev. D 14, 2460-2473 (1976), doi:10.1103/PhysRevD.14.2460

[2] S. W. Hawking, "Black hole explosions," Nature, 248, 30-31 (1974), doi:10.1038/248030a0.

[3] Maulik K. Parikh, The Volume of black holes, Phys. Rev. D., 73, 124021, (2006), doi: 10.1103/PhysRevD.73.124021, arXiv: 0508108[hep-th], reportNumber: CU-TP-1134,

[4] Marios Christodoulou and Carlo Rovelli, , How big is a black hole?, Phys. Rev. D., 6(91), 064046, (2015), doi: 10.1103/PhysRevD.91.064046, arXiv: 1411.2854 [gr-qc].

[5] Marios Christodoulou, and Tommaso De Lorenzo, Volume inside old black holes, Phys. Rev. D., 10(94). 104002, (2016), doi: 10.1103/PhysRevD.94.104002, arXiv: 1604.07222 [gr-qc]

[6] Zhang, Baocheng, Entropy in the interior of a black hole and thermodynamics, Phys. Rev. D., 8(92), 081501, (2015), doi: 10.1103/PhysRevD.92.081501, arXiv: 1510.02182[gr-qc].

[7] Ingemar Bengtsson and Emma Jakobsson, Black holes: Their large interiors, Mod. Phys. Lett. A., 30(30), 1550103, (2015), doi: 10.1142/S0217732315501035, arXiv: 1502.01907[gr-qc]

[8] Yen Chin Ong, The Persistence of the Large Volumes in Black Holes, Gen. Rel. Grav., 8(47), 88, (2015), doi: 10.1007/s10714-015-1929-x, arXiv: 1503.08245[gr-qc].

[9] Shan-Zhong Han, and Jian-Zhi Yang, and Xin-Yang Wang and Wen-Biao Liu, The Entropy Inside a Charged Black Hole Under Hawking Radiation, Int. J. Theor. Phys., 11(57), 3429-3435 , (2018), doi: 10.1007/s10773-018-3856-6.

[10] Wang, Xin-Yang and Jiang, Jie and Liu, Wen-Biao, Entropy in the interior of a Kerr black hole, Class. Quant. Grav. 21(35), 215002, (2018), doi: 10.1088/1361-6382/aae276, arXiv: 1803.09649[gr-qc]. 
[11] Ali, Shad and Wang, Xin-Yang and Liu, Wen-Biao, Entropy in a d-dimensional charged black hole, Int. J. Mod. Phys. A., 27(33), 1850159, (2018), doi: 10.1142/S0217751X18501592.

[12] Shad Ali, Wang, Xin-Yang and Liu, Wen-Biao, Entropy Evolution in the Interior Volume of a Charged f(R) Black Hole, Commun. Theor. Phys., 6 (71), 718, (2019), doi: 10.1088/0253-6102/71/6/718.

[13] Jian-Zhi Yang and Liu, Wen-Biao, Entropy in the interior of a higher-dimensional black hole, Phys. Lett. B., 782, 372-374, (2018), doi: 10.1016/j.physletb.2018.05.050.

[14] S. W Hawking, Particle Creation by Black Holes, 1st Oxford Conference on Quantum Gravity Chilton, England, February 15-16, 219-267, (1975).

[15] Clair L. Wyatt, Chapter IV: Black body Radiation, Book: Radiometric Calibration: Theory and Methods, Academic Press, 29-37, (1978), ISBN: 9780127661506, doi: https:/ /doi.org/10.1016/B978-0-12-766150-6.50007-4. 